\title{
THE EXPECTED NUMBER OF EXTREME POINTS OF A RANDOM LINEAR PROGRAM
}

\author{
Sancho E. BERENGUER \\ Instituto de Matematica Pura e Aplicada, Rio de Janeiro, 22460 Brazil
}

\author{
Robert L. SMITH \\ Department of Industrial and Operations Engineering, The University of Michigan, Ann Arbor, \\ MI 48109, USA
}

Received 6 October 1983

Revised manuscript received 24 January 1985

\begin{abstract}
There has been increasing attention recently on average case algorithmic performance measures since worst case measures can be qualitatively quite different. An important characteristic of a linear program, relating to Simplex Method performance, is the number of vertices of the feasible region. We show $2^{n}$ to be an upper bound on the mean number of extreme points of a randomly generated feasible region with arbitrary probability distributions on the constraint matrix and right hand side vector. The only assumption made is that inequality directions are chosen independently in accordance with a series of independent fair coin tosses.
\end{abstract}

Key words: Random linear program, random polytope, extreme points.

Attempts to establish the mean number of extreme points and other topological properties of random linear constraint sets have included distribution dependent approaches (see, for example, Efron (1965), Liebling (1972), Schmidt and Mattheiss $(1977,1980))$ as well as essentially distribution free approaches (May and Smith (1982), Adler and Berenguer (1981), (1983)). Apart from the purely mathematical interest of these models and results, they have proved useful in establishing average case performance of variants of the Simplex Method (Borgwardt (1982), Adler (1983), Saigal (1984), Smale (1983), Todd (1983)), Adler, Karp and Shamir (1982), Adler and Meggido (1983), and Haimovich (1983)).

McMullen (1970) demonstrated that the worst case number of extreme points goes up factorially fast in problem dimension for the class of convex polytopes. Independently, May and Smith (1982) and Adler and Berenguer (1981), using the theory of arrangements of hyperplanes partitioning Euclidean space (Grunbaum (1967), Buck (1943)), established distribution invariant topological results for randomly generated linear constraint sets. They showed that the average number of extreme points for a class of distributions over convex polytopes increases only exponentially fast in problem dimension. They assumed the generating distribution

We would like to thank the Institute of Pure and Applied Mathematics in Rio de Janeiro for supporting the authors' collaboration that led to this paper. 
to be continuous, thus excluding sparsity, integer constraint coefficients, degeneracy, and other characteristics that real world and many randomly generated linear programs are known to possess. It was also assumed that inequality directions were decided on the basis of independent tosses of a fair coin. Retaining only the latter assumption, (now referred to as sign invariance) we show in this paper that for arbitrary distributions, the expected number of extreme points is bounded from above by $2^{n}$ where $n$ is the dimension of the constraint set. Note that this result for random linear programs does not exclude degeneracy.

\section{A model for random linear programs}

We express the constraint sets in the general form $A x \gtreqless b$ where $A$ is an $m \times n$ matrix, $b$ is column $m$-vector and $x$ is a column $n$-vector. The inequality directions are specified by a column $m$-vector $e$, where $e_{i}=0$ or 1 according to whether the $i$ th constraint is $\leqslant$ or $\geqslant$ respectively. A probability distribution can then be specified over the space of all possible polyhedral sets $S=(A, b, e)$ for $n$ and $m$ fixed. Let $F 1, F 2$ and $F 3$ be the marginal distributions on $A, b$ and $e$ respectively. In particular define $F 3$ by the $m$-tuple $\left(p_{1}, p_{2}, \ldots, p_{m}\right)$ where $p_{i}=P\left(e_{i}=1\right)$ and $e_{1}, e_{2}, \ldots, e_{m}$ are assumed independent. Most of the occurrences of $S$ correspond to empty polyhedral sets. We shall, however, be concerned with conditional properties of polyhedral sets so generated given that they are nonempty. After randomly generating the constraint set, we can obtain a region of dimension $0,1, \ldots$, or $n$. Let $E_{k}(m, n)$ denote the expected number of extreme points of a generated region of dimension $k$. Then the expected number of extreme points of a generated region,

$$
E(m, n)=\sum_{k=0}^{n} E_{k}(m, n) p(k),
$$

where $p(k)$ is the probability of generating a region of dimension $k$.

If $E_{k}(m, k) \leqslant 2^{k}$ we would then have

$$
E(m, n)=\sum_{k=0}^{n} E_{k}(m, k) p(k) \leqslant \sum_{k=0}^{n} 2^{k} p(k) \leqslant 2^{n} \sum_{k=0}^{n} p(k)=2^{n} .
$$

It suffices to show, without loss of generality, that

$$
E_{n}(m, n) \leqslant 2^{n}
$$

(we would clearly have that $E_{k}(m, n) \leqslant 2^{k}$ ).

In particular, we are interested in

$E(n, m)=$ the expected number of extreme points of a nonempty polyhedral set formed by $m$ constraints in $\mathbb{R}^{n}$ according to the model above. 
We shall impose the following restrictions. We require that $p_{i}=\frac{1}{2}$ for $i=1, \ldots, m$ in the $m$-tuple for $F 3$; this is the crucial symmetry assumption of the main results of May and Smith (1982) and Adler and Berenguer (1981, 1983).

We shall here extend the exponential bound of $2^{n}$ for $E(m, n)$ obtained in May and Smith (1982) and Adler and Berenguer $(1981,1983)$ by weakening the assumptions of $F 1$ and $F 2$ to arbitrary distributions over the constraint set matrix and right hand side vector respectively. Incidently their joint distribution is also allowed to be arbitrary. In keeping the one half symmetry on $F 3$ we retain the assumption that each polyhedral set of the partition of $\mathbb{R}^{n}$ formed by the $m$ hyperplanes is equally likely to occur as the chosen feasible region.

\section{A bound on the expected number of extreme points for an arbitrary number of constraints}

Theorem 1. Let $F 1$ over $A$ and $F 2$ over $b$ be arbitrary and possibly dependent distributions. Let $e_{i}, i=1,2, \ldots, m$, be i.i.d. Bernoulli random variables with $p_{i}=\frac{1}{2}$ for all $i=1, \ldots, m$ where $e$ is independent of $A$ and $b$. Then the expected number of extreme points for the corresponding randomly generated nonempty constraint set of $m$ constraints and $n$ variables, $E(n, m) \leqslant 2^{n}$ for all $m$ and $n$.

Before proving Theorem 1 we need the following results.

Definitions (Winder (1966)). A set of $k$ hyperplanes in $\mathbb{R}^{n}$ all passing through a common point is odd-degenerate if the dimension of their intersection is of different parity than $n-k$. A set of hyperplanes that is not odd-degenerate is termed evendegenerate.

Lemma 1 (Winder (1966)). The number of regions in which $m$ hyperplanes, all passing through some common point, divide $\mathbb{R}^{n}$ is equal to the number of distinct even-degenerate subsets of the given $m$ hyperplanes minus the number of distinct odd-degenerate subsets (the empty subset is included and counted as even-degenerate).

Lemma 2. Suppose a point $p$ is determined by (that is, coincides with the intersection of) the distinct hyperplanes $H_{1}^{p}, \ldots, H_{l}^{p}$ in $\mathbb{R}^{n}$. Consider the hyperplane $H \notin\left\{H_{1}^{p}, \ldots, H_{l}^{p}\right\}$, also passing through $p$ and with the property that it does not contain any linear flat of dimension greater than zero which is the intersection of two or more of the $H_{j}^{p}, j=1, \ldots$, l. Let $N_{H}(p)$ be the number of regions (n-dimensional polyhedral sets) formed by the $H_{j}^{p}, j=1, \ldots, l$, and lying entirely in a distinguished half-space of $H$. Then $N_{H}(p)$ is invariant over all $H$ satisfying the property above.

Proof. It is sufficient to show that the number of even-degenerate and odd-degenerate sets of the partition of $\mathbb{R}^{n}$ by $H, H_{1}^{p}, \ldots, H_{l}^{p}$ is invariant to the choice of $H$, so long as it satisfies the property stated above. Then, from Lemma 1 , the number of regions 
in the partition formed by $H_{1}^{p}, \ldots, H_{l}^{p}$ and cut by $H$ is invariant to $H$. The remaining uncut regions are then invariant in number, so that the half of these uncut regions lying wholly in a distinguished half-space is invariant in number.

Let $S \equiv H_{i_{1}}^{p} \cap H_{i_{2}}^{p} \cap \cdots \cap H_{i_{q}}^{p}$, where $H_{i_{j}}^{p} \in\left\{H_{1}^{p}, \ldots, H_{l}^{P}\right\}$. It suffices to show that the parity of $H \cap S$ is invariant with respect to $H$. Let $\operatorname{dim}(S)=k \geqslant n-q$. Clearly, $\operatorname{dim}(H \cap S)=k-1$, for $k>0$, by the property above that $H$ satisfies. Also, $\operatorname{dim}(H \cap$ $S)=0$ for $k=0$; obviously, in this case $H \cap S \equiv S \equiv\{p\}$. Therefore, parity of $(H \cap S)$ is invariant with respect to $H$.

We can therefore simplify the notation substituting $N(p)$ for $N_{H}(p)$.

Lemma 3. Consider a point $p$ determined in $\mathbb{R}^{n}$ by hyperplanes $H_{1}^{p}, \ldots, H_{m}^{p}$. Let $C(p)$ be the total number of regions formed by $H_{1}^{p}, \ldots, H_{m}^{p}$ around $p$. Then $C(p) \leqslant 2^{n} N(p)$.

Proof. It can be easily seen that there must exist $n$ linearly independent hyperplanes out of $\left\{H_{1}^{p}, \ldots, H_{m}^{p}\right\}$ which suffice to determine $p$. Without loss of generality denote these $n$ hyperplanes $H_{1}^{p}, \ldots, H_{n}^{p}$. Now consider the $2^{n}$ orthants determined by $H_{1}^{p}, \ldots, H_{n}^{p}$ and number them 1 through $2^{n}$. Let the number of regions in orthant $i$ be $C_{i}\left(i=1, \ldots, 2^{n}\right)$. Then there clearly exists a hyperplane $H_{i}$, satisfying the property of Lemma 2 , a half-space of which wholly contains the $i$ th orthant. By Lemma $2, C_{i} \leqslant N(p)\left(i=1, \ldots, 2^{n}\right)$ since every region in the orthant entirely lies in a distinguished half-space of $H_{i}$. Hence it follows that

$$
C(p)=\sum_{i=1}^{2^{n}} C_{i} \leqslant \sum_{i=1}^{2^{n}} N(p)=2^{n} N(p) .
$$

Now, consider the partition of $\mathbb{R}^{n}$ by the $m$ hyperplanes of the constraint set $A x \leqq b$. We shall assume throughout that the $m$ hyperplanes are distinct, for otherwise we simply embed the problem in the corresponding lower dimensional subspace. Let $p_{r}, r=1,2, \ldots, k$ be the vertices of the partition ( $k$ is the number of vertices of the partition, $\left.k \leqslant\left(\begin{array}{c}m \\ n\end{array}\right)\right)$. Let $H$ be a hyperplane in $\mathbb{R}^{n}, H_{+}$a distinguished half-space of $H$ and $H^{P_{r}}$ and $H_{+}^{P_{r}}$ the hyperplane parallel to $H$ passing through $p_{r}$ and the half-space corresponding to $H^{P_{r}}$ respectively. Since the number of hyperplanes in the partition is finite, we can find $H$ such that $H^{P_{r}}$ together with the hyperplanes $H_{j}^{P_{r}}$ that determine $p_{r}$ on the partition $\left(j=1, \ldots, l_{r}\right)$ have the property of Lemma 2. ${ }^{1}$ By Lemma 2, exactly $N\left(p_{r}\right)$ regions around $p_{r}$ lie entirely in $H_{+}^{P_{r}}$, and we associate these regions to $p_{r}$ Also clearly no region $R$ of the partition can be associated to two distinct points, say $p_{s}$ and $p_{t}$ where $s \neq t .^{2}$ We can then state the following lemma whose proof is straightforward.

${ }^{1}$ Let $a^{i}$ be the $i$ th row of $A$ corresponding to a normal of the $i$ th hyperplane for $i=1, \ldots, m$. Since the union of the spans of all subsets of $a^{1}, \ldots, a^{m}$ with $n-1$ vectors or less cannot be all of $\mathbb{R}^{n}$, there must exist a vector $a \in \mathbb{R}^{n}$ that is not in the span of any subset of $a^{1}, \ldots, a^{m}$ of $n-1$ vectors or less. Hence the solution set of $a x=0$ (representing hyperplane $H$ ) cannot contain the intersection of the solution sets of any $n-1$ or less of the equations $a^{1} x=0, \ldots, a^{m} x=0$.

${ }^{2}$ Suppose $R \subseteq H_{+}^{p_{s}} \cap H_{+}^{p_{t}}$ without loss of generality, $H_{+}^{p_{s}} \subseteq H_{+}^{p_{r}}$ and $H_{+}^{p_{t}} \subsetneq H_{+}^{p_{s}}$, so that $p_{t} \notin H_{+}^{p_{s}}$. But $p_{t} \in R$ and $R \subseteq H_{+}^{p_{s}}$. Contradiction. 
Lemma 4. Consider the partition of space formed by $m$ arbitrary hyperplanes in $\mathbb{R}^{n}$. Then the total number of regions in the partition $N \geqslant \sum_{r=1}^{k} N\left(p_{r}\right)$ where $p_{1}, \ldots, p_{k}$ are the points formed by the partition (the bound on the right is understood to be zero if there are no points).

We can now easily prove Theorem 1, using the lemmas above.

Proof of Theorem 1. $E(m, n)=$ the expected number of extreme points for a randomly selected region of the partition $=$ average number of extreme points of regions of the partition $=\sum_{r=1}^{k} C\left(p_{r}\right) / N \leqslant \sum_{r=1}^{k} 2^{n} N\left(p_{r}\right) / \sum_{r=1}^{k} N\left(p_{r}\right)=2^{n}$.

\section{Concluding remarks}

The bound of $2^{n}$ for the expected number of extreme points established in Theorem 1 is approached in the limit as the number of constraints increases for absolutely continuous independent distributions $F 1$ and $F 2$ as shown in May and Smith (1982), Adler and Berenguer (1981). We suspect although we have not shown that all other topological properties are also bounded by those characteristic of an $n$-dimensional hypercube. Also since the proofs do not directly exploit the linearity of the varieties partitioning space, the same results can be expected for a wide class of nonlinear programs. The more interesting direction would be a weakening of the symmetry assumption on the inequality directions. Unfortunately, such a move would introduce strong distributional dependence of average topological properties on the particular choice for $F 1$ and $F 2$ as well as on their particular form of dependency.

\section{References}

[1] I. Adler, "The expected number of pivots needed to solve parametric linear programs and the efficiency of the self-dual simplex method", Working Paper, Industrial Engineering and Operations Research Department, University of California (Berkeley, CA, 1983).

[2] I. Adler and S.E. Berenguer, "Random linear programs", Operations Research Center Report No. 81-4, University of California (Berkeley, CA, 1981).

[3] I. Adler and S.E. Berenguer, "Generating random linear programs", unpublished manuscript, Industrial Engineering and Operations Research Department, University of California (Berkeley, CA, 1983), submitted to Mathematical Programming.

[4] I. Adler, R.M. Karp and R. Shamir, "A simplex variant solving an $m \times d$ linear program in expected number of pivots depending on $d$ only", Report UCB CSD 83/157, Computer Science Division, University of California (Berkeley, CA, December, 1983).

[5] I. Adler and N. Meggido, "A simplex algorithm whose average number of steps is bounded between two quadratic functions of the smaller dimension", unpublished manuscript (December, 1983).

[6] K.H. Borgwardt, "Some distribution-independent results about the asymptotic order of the average number of pivot steps of the simplex method", Mathematics of Operations Research 7 (1982) 441-462.

[7] R.C. Buck, "Partition of space", American Mathematical Monthly 50 (1943) 541-544.

[8] B. Efron, "The convex hull to a random set of points", Biometrika 52 (1965) 331.

[9] B. Grunbaum, Convex polytopes (John Wiley \& Sons, New York, 1967). 
[10] M. Haimovich, "The simplex algorithm is very good! On the expected number of steps and related properties of random linear programs", unpublished manuscript, Columbia University (New York, April 1983).

[11] T.M. Liebling, "On the number of iterations of the simplex method", Methods of Operations Research XVII 17 (1972) 248-264.

[12] J.H. May and R.L. Smith, "Random polytopes: Their definition, generation, and aggregate properties", Mathematical Programming 24 (1982) 39-54.

[13] P. McMullen, "The maximum number of faces of a convex polytope", Mathematika 17 (1970) $179-184$.

[14] R. Saigal, "A variant that solves random convex quadratic programs in average steps bounded by a quadratic function of size", Working Paper, Department of Industrial Engineering, Northwestern University (Evanston, IL, 1984).

[15] B.K. Schmidt and T. H. Mattheiss, "The probability that a random polytope is bounded", Mathematics of Operations Research 2 (1977) 292-296.

[16] B.K. Schmidt and T.H. Mattheiss, "Computational results on an algorithm for finding all vertices of a polytope", Mathematical Programming 18 (1980), 308-329.

[17] S. Smale, "The problem of the average speed of the simplex method", in: A. Bachem, M. Grötschel, and B. Korte, eds., Mathematical Programming Bonn 1982 (Springer-Verlag, Berlin, 1983) pp. $530-539$.

[18] M.J. Todd, "Polynomial expected behaviour of a pivoting algorithm for linear complementarity and linear programming problems", Technical Report No. 595, School of Operations Research and Industrial Engineering, Cornell University (Ithaca, NY, 1983).

[19] R.O. Winder, "Partitions of $N$-space by hyperplanes", Journal of the Society for Industrial and Applied Mathematics 14 (1966) 811-819. 